\title{
Immunolabeling of "Nucleoli" in Mouse Fully-Grown Oocytes and One-Cell Embryos is Dependent on Upstream Molecular Fixatives
}

\author{
Elena A. Lavrentyeva ${ }^{1,2}$, Kseniya V. Shishova1, Vladimir S. Mikoyan", \\ Yaroslav M. Stanishevskiy ${ }^{2}$, Olga V. Zatsepina ${ }^{1^{*}}$ \\ 1, *Shemyakin-Ovchinnikov Institute of Bioorganic Chemistry, Russian Academy of Sciences, \\ Moscow, Russia \\ ${ }^{2}$ RUDN University, Moscow, Russia
}

\begin{abstract}
Rather than normal nucleoli, mammalian fully-grown (germinal vesicle, GV) oocytes and one-cell embryos (zygotes) contain intranuclear inclusions called "nucleolus-like bodies, NLBs" and "nucleolar precursor bodies, NPBs", respectively. Both entities remain almost inaccessible to various antibodies following standard procedure of immunofluorescence labeling that makes their protein composition and putative functions vague. Here we examine effects of molecular fixatives on the immunodetection of key nucleolar proteins in mouse NLBs and NPBs following different protocols of their fixation and post-fixation treatment. Our results show that the most impoverished for nucleolar proteins are zygotic NPBs, while only NSN-type NLBs contain all key nucleolar protein examined, including an rRNA processing factor SURF6/Rrp14. These observations support the idea that "nucleoli" of GV oocytes and zygotes have different capacities for ribosome biogenesis. The NSN-type NLBs can be involved in all nucleolar steps of ribosome production, including rDNA transcription, rRNA processing and pre-ribosome assembly. The NLBs of more mature SN-oocytes may only be capable of pre-ribosome assembly, whereas zygotic NPBs are, most likely, excluded from ribosome production.
\end{abstract}

Keywords: nucleolar proteins, NLBs, NPBs, ribosome biogenesis, mouse.

Abbreviations: GV, germinal vesicle; NLB, nucleolus-like body; NPB, nucleolar precursor body; NSN-oocyte, non-surrounded oocyte; SN-oocyte, surrounded oocyte; PFA, paraformaldehyde; PMSG, pregnant mare serum gonadotropin; hCG, human chorionic gonadotropin; dbcAMP, dibutyryl-cAMP; PFA, paraformaldehyde (a polymeric form of formaldehyde); PBS, phosphate-buffered saline; DAPI, 4',6-diamidino-2-phenylindole.

\section{INTRODUCTION}

Unlike somatic cells, mammalian pre-ovulation oocytes (also known as fully-grown or germinal vesicle (GV) oocytes) are devoid of normal (tripartite) nucleoli. Instead, they possess large, up to 10 microns in diameter, intranuclear entities - nucleolus-like bodies (NLBs) which substitute normal nucleoli present in younger oocytes. Based on large-scale chromatin configuration as well as transcriptional activity and meiotic competence, two main types of GV oocytes can be distinguished $[16,23]$. In the mouse, GV oocytes, where NLBs contact with distinct blocks of condensed chromatin (chromocenters), are called non-surrounded (NSN) oocytes, while the oocytes, which contain NLBs surrounded by a prominent layer of condensed chromatin, are called surrounded (SN) GV oocytes. The oocytes of both types are capable of gene expression [16]. However, the rRNA synthesis has only been described in the NSN-type oocytes [1, 20, 24], whereas the SN-type oocytes possess better developmental capacities [18].

The nucleolar precursor bodies (NPBs) are formed in zygotic pronuclei shortly after fertilization and are remarkably similar to NLBs of GV oocytes in morphology [6, 7]. The NPBs are optically dense bodies of 1-10 microns in diameter, which do not incorporate precursors of RNA or DNA synthesis and appear as dark (unstained) zones under standard immunolabelling procedures e.g., [5, 19, 25]. It is not surprising therefore that for a long time NLBs of GV oocytes and NPBs of zygotic embryos were considered as organelles with similar or even alike composition and functions. However, recent experiments showed that microsurgical removal of NPBs, unlike the removal of NLBs, does not prevent assembly of the functional nucleoli and does not affect normal development of multi-cell mouse embryos [15]. These observations strongly support the idea that NPBs and NLBs have different molecular composition and may play different roles. 
It is worth mentioning that the limited number of oocytes and embryos that can be obtained from a mammal ( 30 from a mouse) and the requirement of laborious microsurgical isolation of NPBs and NLBs preclude their study by modern mass-spectrometry methods. Taking into account the total protein content in mouse NLBs (about $1.6 \mathrm{ng} / \mathrm{NLB}$ [8], one proteome analysis may require isolation of over 500 oocytes and zygotic "nucleoli", which is technically very difficult to achieve in the mouse and almost impossible in other mammals, including humans. For this reason, immunocytochemical approaches employing specific antibodies has remained the main approach used to examine protein composition of NLBs and NPBs. However, traditional protocols applied in immunolabeling studies require modification(s) because under standard procedure of oocyte and zygote fixation with formaldehyde (a monomeric form of paraformaldehyde (PFA) in aqueous solutions), the NLB and NPB content remains almost inaccessible for anti-nucleolar antibodies [19, 24, 25].

The present study was conducted to analyze the effects of molecular fixatives on the accessibility of key nucleolar proteins in NLBs of mouse GV oocytes and in NPBs of zygotic embryos. Such an analysis should help specify the functions of these bodies in mammalian oo- and embryogenesis and clarify their putative implication in ribosome biogenesis. Taking into account the importance of fixation procedure in immunocytochemistry [14], the conventional (control) and two modified protocols were employed. In the control experiments, oocytes and embryos were conventionally fixed with PFA/formaldehyde, an aldehyde fixative that well preserves cell morphology but forms additional bonds inside and between protein molecules [4, 12]. The second group of experiments included oocytes and zygotes fixed with PFA/formaldehyde and then subjected to mild proteinase $\mathrm{K}$ digestion that increases accessibility of nucleolar proteins to specific antibodies in somatic cells [22]. In the third group of experiments, oocytes and embryos were fixed with $70 \%$ ethanol, a coagulant fixative that poorly preserves cell morphology but, unlike formaldehyde, removes weakly bound proteins from their native locations [2,14]. Our results show that neither of the nucleolar protein examined were detected inside NLBs or NPBs in PFA/formaldehyde-fixed oocytes and embryos, although most of these proteins (e.g., UBF, fibrillarin, NPM1, nucleolin, RPL26 and RPS10) became detectable inside NLBs and NPBs following both modified protocols. Nevertheless, some proteins (e.g., SURF6/Rrp14) could be detected in NLBs only in 70\% ethanol-fixed oocytes. These observations support the conclusion that a negative immunolabeling of NLBs and NPBs in formaldehydefixed and proteinase K-treated oocytes (zygotes) should be verified using ethanol-fixed cells. Our results also favor the idea that mouse NLBs and NPBs differ in the repertoire of nucleolar proteins and therefore have different capacities to make ribosomes. NLBs of NSN-type GV oocytes can be involved in all nucleolar steps of ribosome biogenesis, including rDNA transcription, rRNA processing and pre-ribosome assembly. The NLBs of more competent SN-oocytes may only be able to assemble and export ribosome subunits, while zygotic NPBs are most likely excluded from the major steps of ribosome production.

\section{MATERIAL AND METHODS}

\subsection{Animals}

Female and male C57B1/6 mice were purchased from the Pushchino Nursery of Laboratory Animals (Pushchino, Russia). The animals were kept under pathogen-free conditions with a free access to tap water and standard chow. All manipulations with animals were performed according to the local law and the principles of good laboratory animal care.

\subsection{Collection of Oocytes and Embryos}

Four-to-eight-week old females were used in all experiments. To obtain GV oocytes, females were injected with 7 IU PMSG (pregnant mare's serum gonadotropin) (Sigma-Aldrich, USA) and sacrificed 46-48 h later. Oocytes were collected from ovaries by gentle puncturing of ova in M2 medium (Sigma-Aldrich) supplemented with $100 \mu \mathrm{g} / \mathrm{ml}$ dibutyryl-cAMP (dbcAMP, Santa Cruz Biotechnology Inc., USA) to prevent resumption of meiosis. Overall, over 300 oocytes were examined. To obtain zygotes, the females were injected with 7 IU PMSG (Sigma-Aldrich) followed by 7 IU hCG (human chorionic gonadotropin) (Sigma-Aldrich, USA) 46-48 h later and caged with males. The females were sacrificed, and zygotes were collected from the ampullae 21-23 hours after hCG injection. Overall, over 150 zygotes were examined. All manipulations with oocytes and embryos were carried out under a Stemi2000 stereomicroscope (Carl Zeiss, Germany) using Stripper mechanical pipettes (Origio, USA) with the inner diameter $135 \mu \mathrm{m}$. 
To determine the type of GV oocytes destined for fixation with $70 \%$ ethanol, a fixative that can deteriorate large-scale chromatin configuration (Tan et al., 2009), isolated oocytes were placed in 50$100 \mu \mathrm{m}$ drops of $1 \mu \mathrm{g} / \mathrm{ml}$ Hoechst 33342 (Life Technologies, USA) dissolved in M2 medium containing $100 \mu \mathrm{g} / \mathrm{ml} \mathrm{dbcAMP}$. Oocytes were quickly analyzed under an Axiovert200 epifluorescence microscope (Carl Zeiss) equipped with a Plan Apochromat phase contrast $\times 20 / 0.8$ numerical aperture objective, and divided into the NSN-type and the SN-type oocytes, which then were separately fixed and processed for immunolabeling.

\subsection{Fixation of Oocytes and Embryos}

Oocytes and embryos were fixed and treated using the following protocols:

(1) Standard (control) protocol [4]: oocytes and embryos were fixed with 3\% paraformaldehyde (PFA, MP Biomedical, Inc., France) in phosphate-buffered saline (PBS, $140 \mathrm{mM} \mathrm{NaCl}, 2.7 \mathrm{mM} \mathrm{KCl}$, $1.5 \mathrm{mM} \mathrm{KH} 2 \mathrm{PO}_{4}, 8.1 \mathrm{mM} \mathrm{Na}_{2} \mathrm{HPO}_{4}, \mathrm{pH} 7.2$ ) for 30-35 min at room temperature. The cells were washed with PBS $(2 \times 5 \mathrm{~min})$, treated with $0.2 \%$ Triton-X100 in PBS $\left(20 \mathrm{~min}, 4^{\circ} \mathrm{C}\right)$ and washed with PBS $(3 \times 5 \mathrm{~min})$.

(2) Modified protocol \#1: oocytes and embryos were fixed with PFA and treated with Triton X-100 as described above. Then, they were exposed to $1 \mu \mathrm{g} / \mathrm{ml}$ proteinase K (Sigma-Aldrich) in PBS for 30-40 $\mathrm{min}$ (in case of oocytes) and for 15-20 min (in case of zygotes) at room temperature. For zygotes, the duration of treatment was reduced because NPBs appeared to be more sensitive to proteinase K, and a longer treatment with the enzyme led to their complete disappearance. For the control, oocytes (zygotes) were incubated in PBS alone for the same time. After proteinase K, the cells were thoroughly washed with PBS (5×5 min).

(3) Modified protocol \#2: oocytes and embryos were fixed with 70\% ethanol for 25-30 min at room temperature, washed with PBS $\left(2 \times 5\right.$ min), treated with $0.2 \%$ Triton $\mathrm{X}-100$ in PBS $\left(10 \mathrm{~min}, 4^{\circ} \mathrm{C}\right)$ and washed with PBS ( $3 \times 5 \mathrm{~min})$.

\subsection{Immunolabelling}

After the last washing with PBS, oocytes (embryos) were incubated with antibodies to the following nucleolar proteins: the specific RNA polymerase I transcription cofactor UBF [26]; the main early rRNA processing factor fibrillarin (Abcam, ab5821); the rRNA processing factors NPM1 (SigmaAldrich, B0556), nucleolin (Abcam, ab70493) and SURF6/Rrp14 (Romanova et al., 2006a) or the ribosomal proteins RPL26 (Abcam, ab59567) and RPS10 (Abcam, ab151550) diluted in PBS as indicated in Table 1. Oocytes (embryos) were then washed with PBS $(3 \times 10 \mathrm{~min})$, and incubated with a FITC-conjugated goat antibody against human IgG (Sigma-Aldrich, F3512) to detect UBF, an Alexa Fluor ${ }^{\circledR}$ 488-conjugated goat antibody against rabbit IgG (Molecular probes Inc., A-11034) to detect nucleolin, RPL26, RPS10, fibrillarin and SURF6, or with an Alexa Fluor ${ }^{\circledR}$ 488-conjugated goat antibody recognizing mouse IgG (Molecular probes, Inc., A11029) to detect NPM1. Before mounting in DAPI/antifade medium (MetaSystems, Germany), chromatin was stained with DAPI (4',6diamidino-2-phenylindole, $1 \mu \mathrm{g} / \mathrm{ml}$ ) for $10 \mathrm{~min}$ at room temperature. The conjugates were diluted in PBS to appropriate working concentrations following recommendations of the manufactures. Incubation with all antibodies continued for 45-60 min at room temperature in the dark. For each experimental point (p. 2.3), 8-10 oocytes of each type and 9-11 zygotes were stained with every antibody (Table 1) to treat the data statistically.

\subsection{Confocal Laser Scanning Microscopy}

Eight-bit images of single optical sections were acquired with a DuoScanMeta LSM510 confocal laser scanning microscope (Carl Zeiss, Germany) using a Plan-Apochromat 63×/1.40 numerical aperture immersion lens.

Measuring the fluorescence intensity per pixel inside NLBs comparatively to the background fluorescence, including that outside oocytes, in the nucleoplasm and the cytoplasm, was performed with a free access Image $\mathrm{J}$ software (version 1.47; http://imagej.nih.gov/ij/download.html).

\section{RESULTS AND DISCUSSION}

\subsection{Immunolabeling of Oocytes and Embryos}

Many authors paid attention to the surprising fact that NLBs and NPBs remained unstained after the standard fixation of mammalian oocytes and zygotes with PFA/formaldehyde followed by immunolabeling with various anti-nucleolar antibodies. The nucleolar proteins which have not been 
detected within NLBs and NPBs include the RNA polymerase transcription cofactor UBF and the rRNA processing factors fibrillarin, NPM1/B23, nucleolin/C23 and SURF6/Rrp14 [5, 9, 19, 24, 25]. Figure 1 illustrates typical patterns of immunolabeling of the control, i.e. conventionally processed, oocytes (Fig. 1a, b) and zygotes (Fig. 1c, d) for UBF. Neither in the NSN-oocyte (Fig. 1a) nor in the SN-oocyte (Fig. 1b), UBF is detected inside NLBs, although in oocytes of both types, dot-like UBFsignals are clearly seen at the NLB surface. By contrast, UBF becomes clearly visible within NLBs of PFA-fixed oocytes after a mild treatment with proteinase K (Fig. 1e, f). It is important to note that the distribution of UBF within NLBs of the NSN- and SN-oocytes is different: in the NSN-type NLBs UBF forms discrete foci typical for normal nucleoli (Fig. 1e), whereas in the SN-type NLBs it is distributed uniformly, i.e. atypically (Fig. 1f). The immunolabeling pattern of GV oocytes fixed with $70 \%$ ethanol (Fig. 1i, j) was similar to that in PFA-fixed/proteinase K-treated oocytes (Fig. 1e, f), although the intensity of UBF labeling inside SN-type NLBs becomes weaker compared to the NSNtype oocytes (Fig. 1j versus Fig. 1f). This observation supports a conclusion that UBF homogenously distributed over the SN-type NLBs, unlike the "discreet" UBF inside the NSN-type NLBs (Fig. 1e, i), is a weakly-bound protein that becomes easily extracted in the presence of ethanol. Unlike in oocytes, we failed to detect UBF inside or at the surface of NPBs in the control (Fig. 1c, d), proteinase Ktreated (Fig. 1g, h) or ethanol-fixed (Fig. 1k, l) zygotes. These observations permit to assume that UBF is absent from "nucleoli" of one-cell embryos, is in the active state in the NSN-type NLBs but is is the dormant (inactive) state within the SN-type NLBs.

In order to examine the effects of fixation and post-fixation procedures on immunodetection of key nucleolar rRNA processing factors, we stained oocytes and zygotes with antibodies recognizing NPM1/B23, nucleolin/C23, fibrillarin and SURF6/Rrp14 (Table 1). The distribution of NPM1 and nucleolin turned out to be similar, and therefore detailed patterns of immunolabeling is illustrated only for nucleolin (Fig. 2). In control oocytes (Fig. 2 a, b) as well as in control zygotes (Fig. 2c, d), nucleolin was visible in the nucleoplasm but was not detectable inside NLBs (Fig. 2a, b) or NPBs (Fig. 2c, d). However, proteinase K treatment clearly revealed nucleolin inside both NLBs (Fig. 2e, f) and NPBs (Fig. 2g, h). Positive anti-nucleolin labeling of NLBs and NPBs was also observed in oocytes (Fig. 2i, j) and zygotes (Fig. 2k, 1) after their fixation with 70\% ethanol. Some representative oocytes and zygotes immunolabeled for NPM1 are shown in Fig. 3, where (a-c) are the controls (i.e., cells only fixed with PFA), (i) is the NSN-type and (l) is the SN-type oocytes fixed with $70 \%$ ethanol. In the control oocytes of both types (NSN is shown in Fig. 3a, and SN in Fig. 3b) as well as in zygotic NPBs (Fig. 3c) the NPM1 is seen only in the nucleoplasm. However, in ethanolfixed oocytes (Fig. 3i, 1) and zygotes (data not shown), the NPM1 can be observed inside NLBs and NPBs. A similar tendency was also seen when oocytes and zygotes were processed for immunolabeling with an anti-fibrillarin antibody. Fibrillarin became clearly seen within NLBs and NPBs only after proteinase $\mathrm{K}$ treatment or in ethanol-fixed cells (not illustrated).

An evolutionary conserved rRNA processing factor SURF6/Rrp14 has been described as a protein absent from NLBs and NPBs in mouse formaldehyde-fixed GV oocytes and one-cell embryos [19]. In the present study, we were also unable to detect SURF6 in the untreated controls (not shown). We also failed to visualize SURF6 inside NLBs and NPBs following proteinase K digestion (Fig. 3d). However, in 70\% ethanol-fixed oocytes SURF6 became clearly visible inside NLBs of the NSN-type (Fig. 3g). Nevertheless, SURF6 was barely detectable in NLBs of the SN-type oocytes (Fig. 3j) and became undetectable inside zygotic NPBs (Fig. 3m).

Antibodies recognizing ribosomal proteins RPS10 and RPL26 were used to examine a putative involvement of NLBs and NPBs in pre-ribosome assembly. As demonstrated in Fig 4, RPS10 was not seen inside control NLBs (Fig. 4a, b) but became visible inside NLBs after oocyte treatment with proteinase K (Fig. 4 e, f). Despite the labeling of NLBs is rather weak, it, nonetheless, exceeds the intensity of fluorescence outside oocytes more than in ten times. In the majority of cases, it was up to $60 \%$ higher than the fluorescence in the surrounding nucleoplasm and the cytoplasm (Fig. 4e, f). A similar immunolabeling pattern was also observed with the anti-RPL26 antibody (not illustrated). It is noteworthy that under optimal conditions RPL26 and RPS10 were clearly detected in all NLBs of the NSN type ( $n=20)$ (Fig. 4e), but only in approximately a half of the SN-type NLBs $(n=30)$ (Fig. 4f). Zygotic NPBs remained unlabeled for RPL26 and RPS10 following all protocols we used (Fig. 4c, d, $\mathrm{g}-\mathrm{j})$.

The effects of fixation on subsequent immunolabeling procedures are summarized in Table 2 . The Table shows that the conventional procedure of fixation of oocyte and embryos with PFA/ formalde- 
hyde [4] is inappropriate for immunodetection of nucleolar proteins inside NLBs and NPBs. By contrast, the majority of proteins assayed were revealed in NLBs and NPBs following a mild digestion of PFA/formaldehyde-fixed cells with proteinase K or after their one-step fixation with $70 \%$ ethanol. However, in some cases (e.g., in the case of the SURF6 protein), a protein of interest can be detected within NLBs only in the ethanol-fixed cells. This indicates that the negative immunolabeling observed in PFA/formaldehyde-fixed cells should be verified using oocytes/embryos fixed with ethanol.

The overall results of immunofluorescence detection of key nucleolar proteins in mouse NLBs and NPBs are illustrated in Fig. 5. They argue in favor of the idea that three nucleolus-related bodies, namely NLBs of the NSN-type GV oocytes, NLBs of the SN-type GV oocytes and NPBs of zygotic embryos, differ in the repertoire of nucleolar proteins. These proteins are known to be markers of the main steps of ribosome biogenesis in normal nucleoli, including rDNA transcription (UBF), rRNA processing (fibrillarin, SURF6/Rrp14, NPM1, nucleolin) and pre-ribosome assembly (RPS10, RPL26) [10]. The "richest" in the nucleolar proteins assayed were NLBs of the NSN-type oocytes, where all the proteins can be detected with the modified protocols (Fig. 5). Moreover, in the NSN-type NLBs the distribution of UBF was reminiscent of that in active nucleoli [11]. Zygotic NPBs were most impoverished for the nucleolar proteins examined, because only fibrillarin, NPM1 and nucleolin were detected in their interior even under optimal conditions (Fig. 5). The SN-type NLBs occupy an intermediate position: they contained the same proteins as the SN-type NLBs but were impoverished for SURF6. Based on these observations, we assume that, like normal nucleoli, NLBs of the NSNtype GV oocytes are involved in all nucleolar steps of ribosome biogenesis, including rDNA transcription, rRNA processing and pre-ribosome assembly. The NLBs of more mature SN-type oocytes can be capable of assembly of ribosome subunits and their export but not of the rDNA transcription, while zygotic NPBs are presumably excluded from ribosome production.

\section{Conclusions}

We conclude that negative immunolabeling of the nucleolus-related bodies present in mammalian oocytes and embryos requires verification with other molecular protocols, including a mild treatment of PFA/formaldehyde-fixed cells with proteinase $\mathrm{K}$ and one-step fixation of cells with $70 \%$ ethanol.

\section{ACKNOWLEDGMENTS}

The study was financed by the Russian Foundation for Basic Research (grant 16-04-01199).

Table1. The list of anti-nucleolar antibodies $(A b)$ used in the study. The Ab stock concentrations and the working dilutions in PBS are indicated.

\begin{tabular}{|l|l|c|}
\hline \multicolumn{1}{|c|}{ Proteins } & \multicolumn{1}{c|}{ Abs used/Ab stock concentration } & Ab dilution \\
\hline UBF & Zatsepina et al.(1993)/not defined & $1: 200$ \\
\hline Fibrillarin & Abcam (ab5821)/1 mg/ml & $1: 200$ \\
\hline NPM1/B23/nucleophosmin & Sigma-Aldrich $(\mathrm{B} 0556) / 0.5 \mathrm{mg} / \mathrm{ml}$ & $1: 200$ \\
\hline Nucleolin/C23 & Abcam $(\mathrm{ab} 70493) / 0.2 \mathrm{mg} / \mathrm{ml}$ & $1: 100$ \\
\hline SURF6/Rrp14 & Romanova et al. $(2006 \mathrm{a}) / \mathrm{not}$ defined & $1: 100$ \\
\hline RPS10 & Abcam $(\mathrm{ab} 151550) / 1.027 \mathrm{mg} / \mathrm{ml}$ & $1: 200$ \\
\hline RPL26 & Abcam $($ ab59567)/1.1 $\mathrm{mg} / \mathrm{ml}$ & $1: 200$ \\
\hline
\end{tabular}

Table2. Immunodetection of key nucleolar proteins in mouse GV oocytes and one cell embryos (zygotes) following different protocols of cell fixation and post-fixation treatment. GV oocytes include the NSN-and SNtype oocytes unless specified.

\begin{tabular}{|c|c|c|c|}
\hline Proteins & PFA/formaldehyde & $\begin{array}{c}\text { PFA/formaldehyde } \\
\text { +proteinase K }\end{array}$ & 70\% ethanol \\
\hline C23, NPM1,fibrillarin* & - GV oocytes, - zygotes & + GV oocytes, + zygotes & + GV oocytes, + zygotes \\
\hline SURF6 & - GV oocytes, - zygotes & - GV oocytes, - zygotes & $\begin{array}{c}\text { + NSN-type oocytes } \\
\text {-/+ SN-type oocytes } \\
\text { - zygotes }\end{array}$ \\
\hline UBF* & - GV oocytes, - zygotes & + GV oocytes*, - zygotes & + GV oocytes, - zygotes \\
\hline RPS10, RPL26 & - GV oocytes & + NSN-type oocytes \\
& - zygotes & $\begin{array}{c}\text { +(50\%)/-(50\%) SN-type } \\
\text { oocytes }\end{array}$ & - zygotes \\
& & - zygotes & \\
\hline
\end{tabular}

+, prominent signals, -/+, weak if any signals, -, no signals 
Elena A. Lavrentyeva et al.

*, a protein has different distribution in NLBs of NSN- and SN-type oocytes

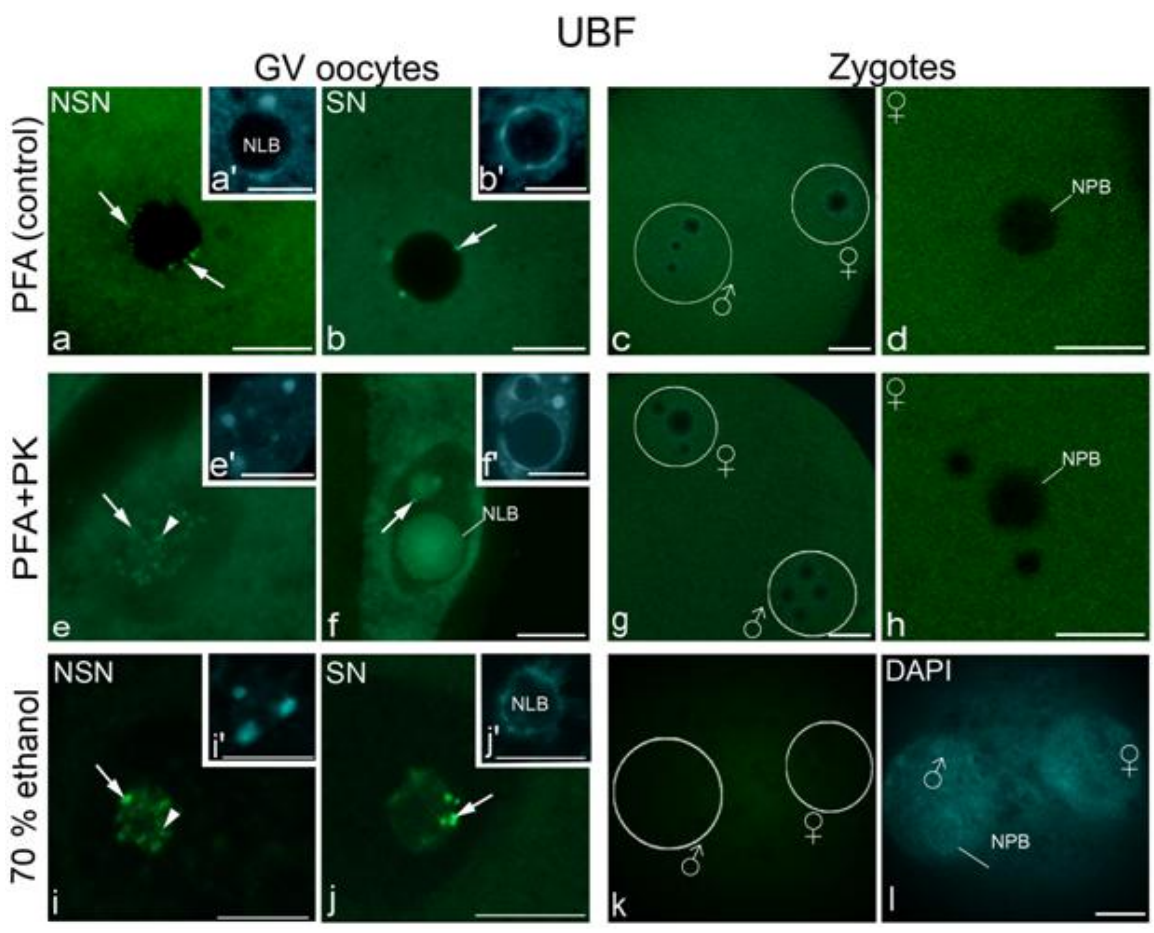

Fig1. Localization of UBF in mouse GV oocytes and zygotes

Immunodetection of UBF, the specific nucleolar transcription cofactor of RNA polymerase I, in GV oocytes (a, b, e, f, i, j) and zygotes (c, d, g, h, k, l) fixed with formaldehyde (PFA, a-d), fixed with formaldehyde and then treated with proteinase K (PFA+PK; e-h), or fixed with $70 \%$ ethanol (i-l). Panels (a', b', e', f', i', j'), large-scale chromatin configuration as seen with DAPI staining. Panels (a, $\left.a^{\prime}\right),\left(e, e^{\prime}\right)$ and (i, i') are the NSN-type oocytes, panels (b, b'), (f, $\left.f^{\prime}\right)$ and $\left(j, j^{\prime}\right)$ are the SN-type oocytes. NLB, nucleolus-like body; NPB, nucleolar precursor body, + , female pronucleus; $\partial^{\lambda}$, male pronucleus. In oocyte images, arrows indicate localization of UBF at the surface of NLBs, and the arrowheads in (e, i) indicate localization of UBF foci inside NLBs. (c, d, g, h, k, l), no UBF signals are detected inside or at the surface of NPBs. Dotted lines define contours of pronuclei. Scale bars, 10 $\mu \mathrm{m}$

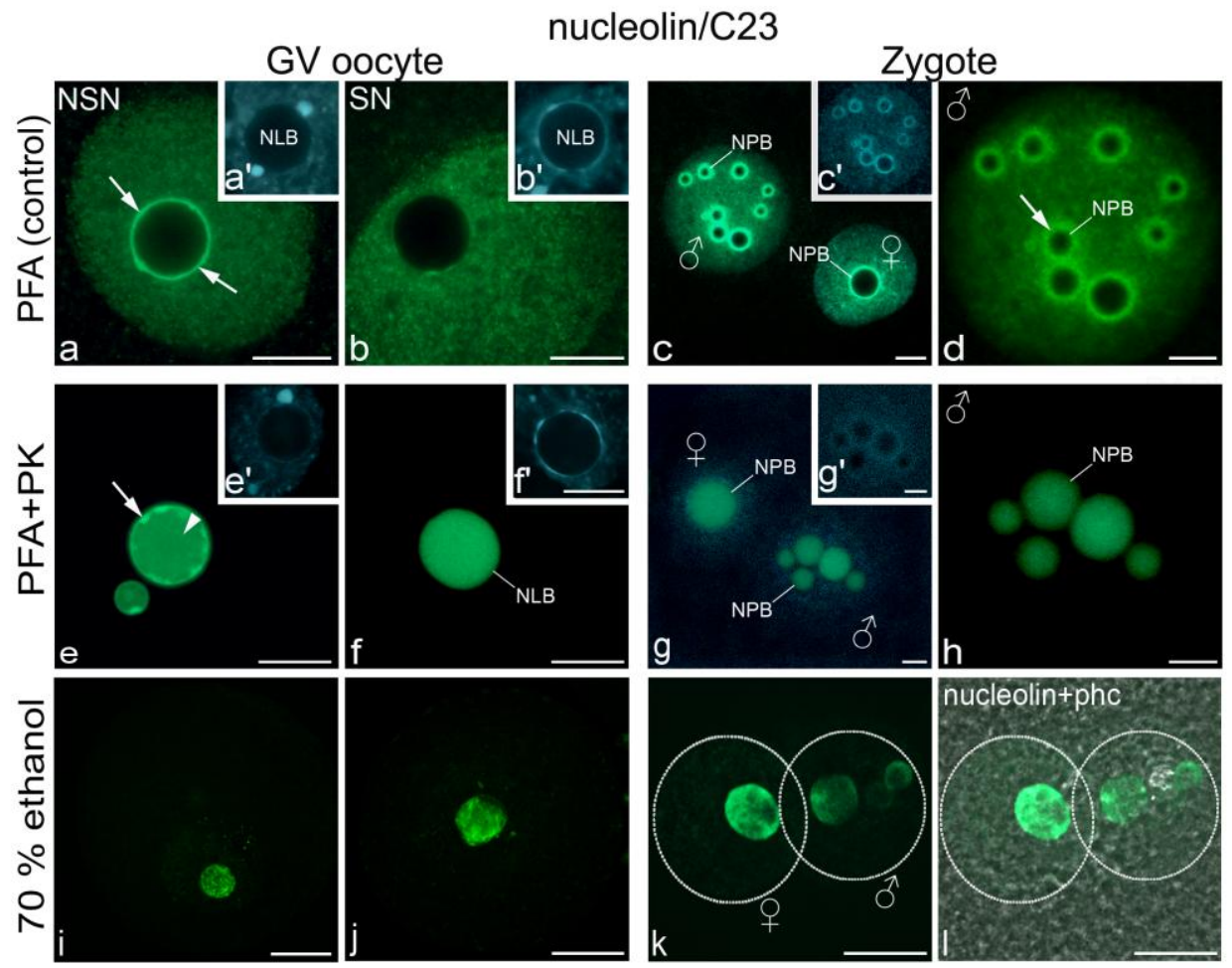


Immunolabeling of "Nucleoli" in Mouse Fully-Grown Oocytes and One-Cell Embryos is Dependent on Upstream Molecular Fixatives

Fig2. Localization of nucleolin in mouse GV oocytes and zygotes

Immunodetection of a multifunctional nucleolar protein nucleolin (C23) in GV oocytes (a, b, e, f, i, j) and zygotes (c, d, g, h, k, l) fixed with formaldehyde (PFA, a-d), fixed with formaldehyde and treated with proteinase K (PFA+PK, e-h), or fixed with $70 \%$ ethanol (i-l). Panels (a', b', c', e', f', g') show DAPI staining of chromatin. Panels (a, a'), (e, e') and (i) are the NSN-type oocytes, panels (b, b'), (f, $\left.\mathrm{f}^{\prime}\right)$ and (j) are the SN-type oocytes, panels (c, c', d), (g, g', h) and $(\mathrm{k}, \mathrm{l})$ are zygotes. NLB, nucleoluslike body; NPB, nucleolar precursor body; + , female pronucleus; $\hat{O}$, male pronucleus. In (a, d, e), arrows indicate nucleolin at the surface of the NLB (NPB), in (e), arrows indicate nucleolin inside NLBs. Dotted lines define contours of pronuclei. Scale bars, $10 \mu \mathrm{m}$.
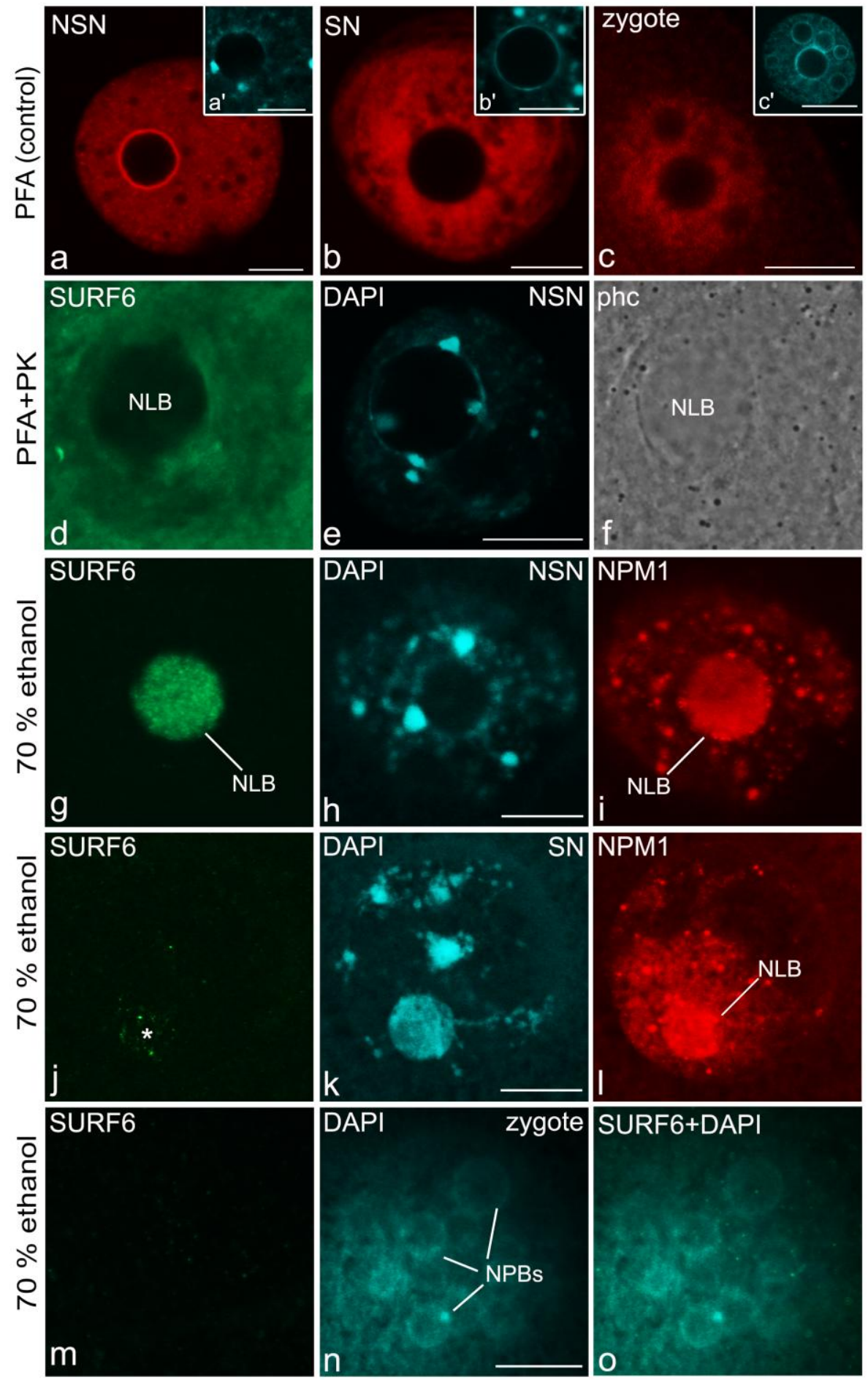

Fig3. Localization of NPM1/B23 and SURF6/Rrp14 in mouse GV oocytes and zygotes 
Elena A. Lavrentyeva et al.

Immunodetection of NPM1/B23 (NPM1; a-c, i, l) and SURF6/Rrp14 (SURF6; d, g, j, m) in oocytes (a-l) and zygotes (m-o) fixed with PFA (control; a-c), fixed with PFA and treated with proteinase K (PFA+PK, d-f) or fixed with 70\% ethanol (g-o). In panels (a', b', c', e, h, k, n) and (o) DAPI staining of chromatin is shown. Panels (a, d-i) are the NSN-type oocytes, (b, j-l) are the SN-type oocytes, and (c, m-o) are zygotes. phc, phase-contrast image of the oocyte shown in (d, e). NLB, nucleolus-like body, NPB, nucleolar precursor body. Scale bars, $10 \mu \mathrm{m}$.

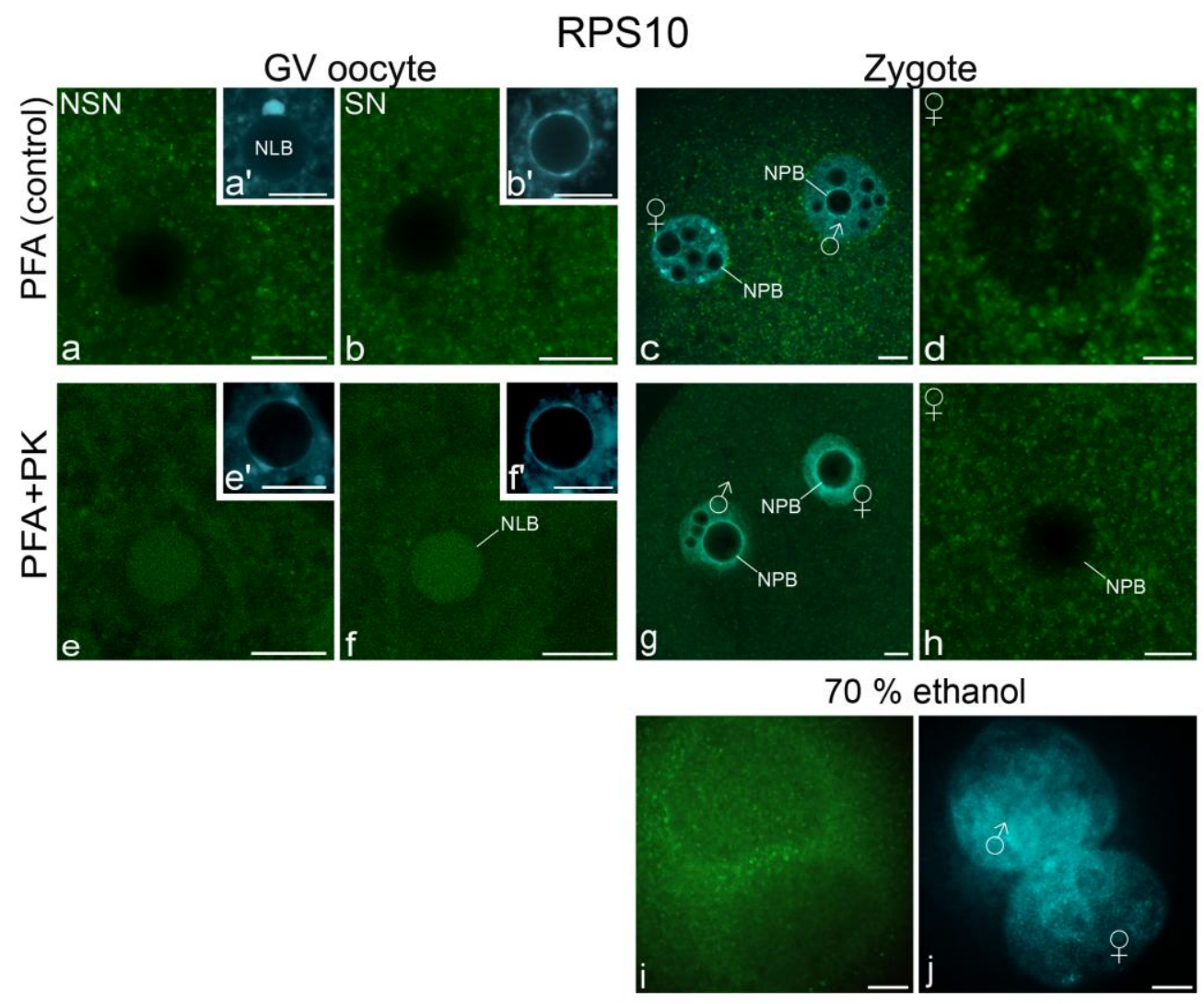

Fig4. Localization of ribosomal protein RPS10 in mouse GV oocytes and zygotes

Immunodetection of RPS10 in oocytes (a, b, e, f) and zygotes (c, d, g, h, i, j) fixed with formaldehyde (PFA, a-d), fixed with formaldehyde and treated with proteinase K (PFA+PK, e-h), or fixed with 70\% ethanol (i, j). Panels (a', b', e', f') and (c, g) illustrate DAPI staining of chromatin. Panels (a, a', e, e') are the NSN-type oocytes, (b, b', f, f') are the SN-type oocytes, and (c, d, g, h, i, j) are zygotes. NLB, nucleolus-like body, NPB, nucleolar precursor body; + , female pronucleus; ${ }^{\lambda}$, male pronucleus. Scale bars, $10 \mu \mathrm{m}$.

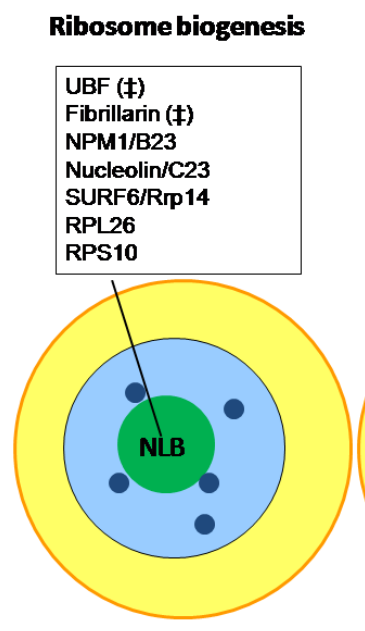

NSN-oocyte

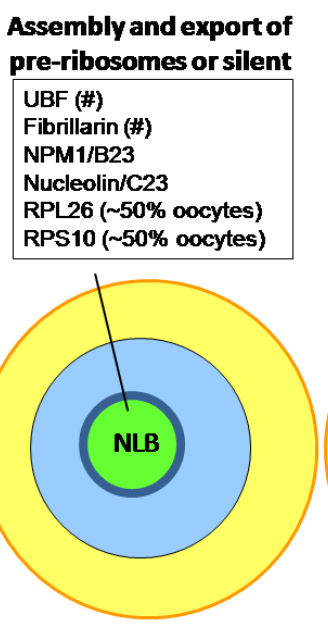

SN-oocyte

\section{Silent in ribosome biogenesis}

Fibrillarin (\#)

NPM1/B23

Nucleolin/C23

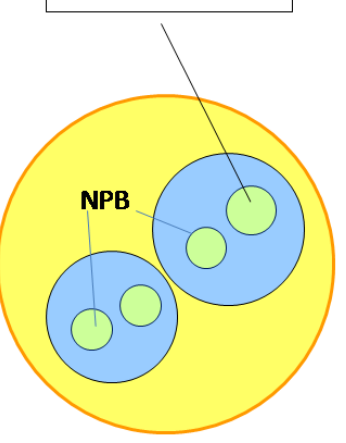

Zygote 
Fig5. Nucleolar proteins detected in mouse NLBs and NPBs under optimal conditions

On the top, putative roles of the NLBs of the NSN- and SN-type oocytes and of zygotic NPBs in ribosome biogenesis are indicated. (\$), normal, the nucleolus-like, localization of a protein, (\#), atypical localization of a protein. The SURF6/Rrp14 protein was unambiguously detected only in the NSN-type NLBs. Ribosomal proteins RPL26 and RPS10 were detected only in 50\% of the SN-type NLBs.

\section{REFERENCES}

[1] C. Bouniol-Baly, L. Hamraoui, J. Guibert, N. Beaujean, M. S. Szöllösi and P. Debey, "Differential transcriptional activity associated to chromatin configuration in fully-grown GV mouse oocytes", Biol. Reprod., vol. 60 pp. 580-587, 1999.

[2] Eltoum, J. Fredenburgh, R. B. Myers and W. E. Grizzle, "Introduction to the theory and practice of fixation of tissues", J. Histotechnol., vol. 24, pp. 173 -190, 2001.

[3] J. Ferreira, M. Carmo-Fonseca, "The biogenesis of the coiled body during early mouse development", Development, vol. 121, no. 2, pp. 601-12, 1995 Feb.

[4] M. Flemr and P. Svoboda, "Ribonucleoprotein localization in mouse oocytes", Methods, vol. 53, pp. 136-141, 2011.

[5] J. E. Fléchon and V. Kopecný, "The nature of the 'nucleolus precursor body' in early preimplantation embryos: a review of fine-structure cytochemical, immunocytochemical and autoradiographic data related to nucleolar function", Zygote, vol. 6, pp. 183-191, 1998.

[6] H. Fulka, H. Kyogoku, O. Zatsepina, A. Langerova A and J. Fulka, "Can nucleoli be markers of developmental potential in human zygotes?” Trends Mol. Med., vol. 21, pp. 663-672, 2015.

[7] H. Fulka and F. Aoki, "Nucleolus precursor bodies and ribosome biogenesis in early mammalian embryos: old theories and new discoveries", Biol. Reprod., vol. 94, pp. 143, 2016.

[8] H. Fulka, S. Martinkova, H. Kyogoku, A. Langerova and J. Fulka, "Production of giant mouse oocyte nucleoli and assessment of their protein content", J. Reprod. Dev., vol. 58, pp. 371-376, 2012.

[9] H. Fulka and A. Langerova, "The maternal nucleolus plays a key role in centromere satellite maintenance during the oocyte to embryo transition", Development, vol. 141, pp.1694-1704, 2014.

[10] K. M. Goudarzi and M. S. Lindström, "Role of ribosomal protein mutations in tumor development", Int. J. Oncol., vol. 48, pp. 1313-1324, 2016.

[11] I. Grummt, "The nucleolus - guardian of cellular homeostasis and genome integrity", Chromosoma, vol. 122, pp. 487-497, 2013.

[12] W. J. Howat, B. A. Wilson, "Tissue fixation and the effect of molecular fixatives on downstream staining procedures", Methods, vol. 70, no. 1, pp. 12-9, 2014 Nov.

[13] B. Kar, B. Liu, Z. Zhou, Y.W. Lam, "Quantitative nucleolar proteomics reveals nuclear reorganization during stress-induced senescence in mouse fibroblast", BMC Cell Biol., vol. 12, no. 33, pp. 1-13, 2011.

[14] A. N. Kuzmin, A. Pliss and P. N. Prasad, "Changes in biomolecular profile in a single nucleolus during cell fixation”, Anal. Chem., vol. 86, pp. 10909-10916, 2014.

[15] H. Kyogoku H, J. Fulka, T. Wakayama and T. Miyano, "De novo formation of nucleoli in developing mouse embryos originating from enucleolated zygotes", Development, vol. 141, pp. 2255-2259, 2014.

[16] A. M. Luciano, F. Franciosi, C. Dieci and V. Lodde, "Changes in large-scale chromatin structure and function during oogenesis: a journey in company with follicular cells", Anim. Reprod. Sci., vol. 149 pp. 3-10, 2014.

[17] A. Magoulas, O. V. Zatsepina, P.W. Jordan, E.G. Jordan, M. Fried, "The SURF-6 protein is a component of the nucleolar matrix and has a high binding capacity for nucleic acids in vitro", Eur. J. Cell Biol., vol. 75, no. 2, pp. 174-83, 1998. 
[18] M. Monti, M. Zanoni, A. Calligaro, M. S. Ko, P. Mauri and C. A. Redi, "Developmental arrest and mouse antral not-surrounded nucleolus oocytes", Biol. Reprod., vol. 88, pp. 1-7, 2013.

[19] L. G. Romanova, M. Anger, O. V. Zatsepina, R. M. Schultz, "Implication of nucleolar protein Surf6 in ribosome biogenesis and preimplantation mouse development", Biol. Reprod., vol. 75, pp. 690-696, 2006a.

[20] K. V. Shishova, E. A. Lavrentyeva, J. W. Dobrucki, O. V. Zatsepina, "Nucleolus-like bodies of fully-grown mouse oocytes contain key nucleolar proteins but are impoverished for rRNA", Dev. Biol., vol. 397, 267-281, 2015.

[21] S. Snaar, K. Wiesmeijer, A. G. Jochemse, H. J. Tanke and R. W. Dirks, "Mutational analysis of fibrillarin and its mobility in living human cells", J. Cell Biol., vol. 151, no. 3, pp. 653-662, 2000.

[22] D. M. Svistunova, Y. R. Musinova, V. Y. Polyakov, E. V. Sheval, "A simple method for the immunocytochemical detection of proteins inside nuclear structures that are inaccessible to specific antibodies", J. Histochem. Cytochem., vol. 60, no. 2, pp. 152-8, 2012 Feb..

[23] J. H. Tan, H. L. Wang, X. S. Sun, Y. Liu, H. S. Sui and J. Zhang, "Chromatin configurations in the germinal vesicle of mammalian oocytes", Mol. Hum. Reprod., vol. 15, pp. 1-9, 2009.

[24] O. V. Zatsepina, C. Bouniol-Baly C, C. Amirand and P. Debey, "Functional and molecular reorganization of the nucleolar apparatus in maturing mouse oocytes", Dev. Biol., vol. 223, pp. 354-370, 2000.

[25] O. V. Zatsepina, C. H. Baly, M. Chebrout and P. Debey, "The step-wise assembly of a functional nucleolus in preimplantation mouse embryos involves the Cajal (coiled) body", Dev. Biol., vol. 253, pp. 66-83, 2003.

[26] O. V. Zatsepina, R. Voit, I. Grummt, H. Spring, M. V. Semenov and M. F. Trendelenburg, "The RNA polymerase I-specific transcription initiation factor UBF is associated with transcriptionally active and inactive ribosomal genes', Chromosoma, vol.102, pp.599-611, 1993. 